\title{
SHORTCUT MULTIPLIER FORMULAS FOR INTERREGIONAL INPUT-OUTPUT MODELS
}

\author{
Joseph L. Katz and Roger L. Burford*
}

\section{Introduction}

One of the most serious practical impediments to the widespread application of regional input-output models for impact analysis is, on the one hand, the extreme time and expense required to construct a detailed survey based model and, on the other hand, the practical inability to develop satisfactory low cost models using secondary data methods. Although these problems have hampered the widespread use of regional input-output models, they have virtually eliminated the interregional input-output models as a viable tool for impact analysis. In fact, the interregional input-output model is still largely a conceptual model which is not often implemented in practice.

The conceptual statement of the interregional input-output model framework dates back at least to the early 1960's (Leontief and Strout (1963)) and has its roots at least as far back as the 1950's (Isard (1951, 1953), Kuenne (1953), Moses (1959), Stein (1958) and Tiebout (1957)) although empirical implementation had to wait until the late 1960's and early 1970's (Garnick (1969), Miernyk (1973), Round (1978, 1979) and Polenske (1970, 1972, 1972a, 1974, 1976, 1978, 1979)). Still, while a great deal of conceptual groundwork of the interregional input-output model has been laid, few empirical examples exist due to the massive data requirements. These data problems include not only the vast quantity of coefficients that must be estimated $\left(k^{2} n^{2}\right.$ coefficients for a $k$ region interregional model with $\mathbf{n}$ industries per region), but also the difficulty firms experience in identifying their industrial sources of purchased inputs and categorizing these expenditures from both within its own region and other specified regions.

HYPOTHESIZED RELATIONSHIP BETWEEN ACCURACY OF MULTIPLIER ESTIMATES AND AMOUNT OF INFORMATION KNOWN FROM COEFFICIENT MATRIX

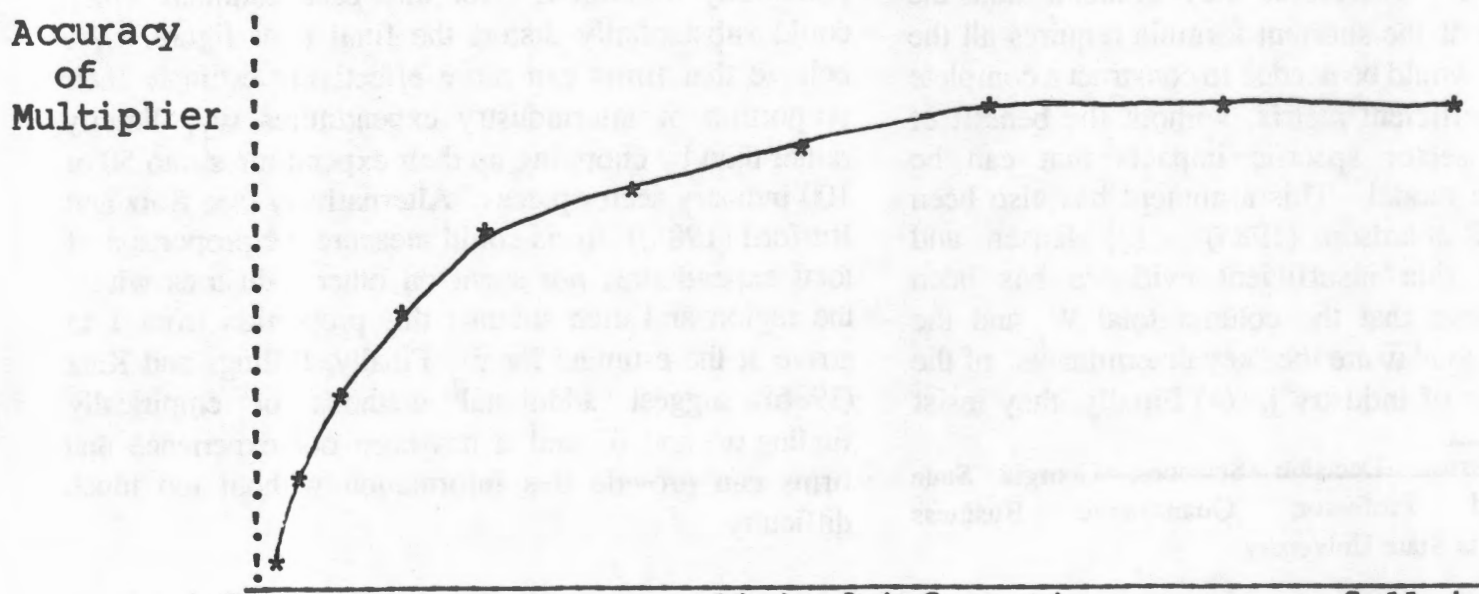


Previously, Burford and Katz (1981) and Katz and Burford (1985) have established the veracity of this representation of the tradeoff curve by first identifying key factors in the regional coefficient matrix that are critical to the formation of the output, income and employment multipliers and then by producing shortcut formulas based on these parameters that closely approximate the multipliers reported in actual inputoutput tables. This paper extends the earlier work on regional models to the estimation of shortcut impact formulas for interregional output, income and employment multipliers.

\section{A Defense of the Shortcut Multiplier Methodology}

Several authors have attacked the utility of the shortcut multiplier formula methodology by raising various arguments that purport to undermine the effectiveness and validity of this procedure. Their major assault is launched against the output multiplier formula in equation (1)

$$
u_{j}=1+\left(w_{j} /(1-\bar{w})\right)
$$

where $w_{j}$ is the proportion of regional interindustry expenditures of firm $j$ (the jth column total of the inputoutput coefficient matrix) and $\bar{W}$ is the average regional interindustry expenditure rate of all firms in the region (the average column total of the input-output coefficient matrix).

Jensen and Hewings (1985) offer four basic complaints against the shortcut multiplier methodology. (1) They argue that shortcut multipliers do not provide as extensive an evaluation of economic impacts as a complete input-output model and so these shortcuts are of little use. (2) They insist that the computation of the column total values $w$. and $\bar{W}$ for the output multiplier formula in equation (1) requires data on the proportion expenditures to each individual industry sector by industry $\mathrm{j}$ and all other industry sectors in the region, namely all the individual coefficients $a_{i j}$. Therefore, they contend that the implementation of the shortcut formula requires all the same detail that would be needed to construct a complete input-output coefficient matrix, without the benefit of the additional sector specific impacts that can be provided by the model. This argument has also been advanced by Richardson (1985). (3) Jensen and Hewings argue that insufficient evidence has been presented to prove that the column total $\mathrm{w}_{j}$ and the average column total $\bar{W}$ are the "key determinants" of the output multiplier of industry j. (4) Finally, they insist

\footnotetext{
*Associate Professor, Decision Sciences, Georgia State University and Professor, Quantitative Business Analysis, Louisiana State University.
}

that the shortcut output multiplier is invalid since it is derived based on a random matrix concept which violates the known structure of input-output matrices.

As to the first point, there is no disagreement with Jensen and Hewings that shortcut multiplier formulas do not provide as much impact information as a complete input-output model. However, the proposition that these shortcut formulas are not useful for their intended purpose is untenable. Shortcut formulas provide a low cost reliable alternative to the measurement of output, income and employment impacts over a particular economic region, which constitutes its main application. Often, to compare the economic effect of different scenarios, planners and analysts need dependable estimates of gross multiplier impacts and generally are unconcerned with breaking down these impacts by specific industry categories (see Jelavich (1984), Katz and Burford (1985), Billings and Katz (1986)). Rarely is a reliable input-output model even available for the particular region under study, and there is strong resistance against devoting an extreme amount of time and money to build one. Although a Rolls Royce costs much more than a Toyota and is furnished with many more extravagant options, both cars provide good reliable transportation and the fact that the Rolls Royce has more features than the Toyota does not in itself imply that Toyotas serve no useful function in the car market.

As to the second point, Jensen and Hewings' contention that the estimation of $w$ and $\vec{W}$ requires the separate measurement of the proporion spent by a firm to each industry sector (the matrix coefficients) is an inefficient and somewhat extreme approach to the problem. The argument that the estimate of the total requires individual measurements of each of its pieces ignores the fact that there is virtually no limit to the number of pieces one could divide up the total figure as a firm could be involved in literally millions of separate economic transactions annually. Furthermore, any attempt to directly measure the individual $a_{i j}$ terms potentially introduces error into each estimate which could substantially distort the final total figure. We believe that firms can more effectively estimate their proportion of interindustry expenditures, $w_{j}$, directly rather than by chopping up their expenditures into 50 or 100 industry sector pieces. Alternatively (see Katz and Burford (1985)), firms could measure the proportion of total expenditures not spent on other industries within the region and then subtract this proportion from 1 to arrive at the estimate for $w_{j}$ Finally, Billings and Katz (1986) suggest additionar methods of empirically finding $w_{j}$ and $\bar{w}$ and it has been our experience that firms can provide this information without too much difficulty. 
As to point three, the evidence suggesting that $w_{j}$ and $\bar{w}$ are the key determinants of the output multiplier of industry $j$ is broad and compelling. As reported by Katz and Burford (1981), the shortcut output multipliers formula, which utilizes only $w_{j}$ and $\bar{w}$ to estimate the output multipliers, has been shown to closely approximate those output multipliers produced by inputoutput models compiled for Utah, Nebraska and West Virginia, with mean absolute percent errors of $1.5 \%$ to $2.5 \%$. Additionally, the accuracy of this formula has been independently verified by Phibbs and Holsman (1981) in tests on other input-output tables. The success of equation (1) on regional input-output models can be credited to the relatively small sizes of $w_{j}$ (usually between 0 and .5). For regional models, empirical tests have revealed that the output multiplier impact for rounds zero and one generally exceed $90 \%$ of the total multiplier impact. As the theoretical multiplier impact for rounds zero and one is $\left(1+w_{j}\right)$ which exactly equals the impact asigned by the shortcut formula for those rounds, thus the shortcut multiplier formula measures the exact impact of the most important rounds and forecasts the impact of latter rounds according the average interindustry expenditure rate for the region, $\bar{w}$. Jensen and Hewings argue that "the role of individual coefficients is of some importance in the matrix." There is no real disagreement with this statement as, in certain cases, extremely large coefficients in a column can significantly inflate or deflate the predicted multiplier. Yet, empirical tests have confirmed that this situation rarely occurs except in the most aggregated models.

As to point four, unlike the usual nonsurvey approaches for estimating input-output coefficients (see Round 1983 for a recent summary of nonsurvey techniques), the "random matrix approach" is a method for generating shortcut multiplier formulas assuming varying degrees of information from the input-output coefficient matrix. Criticisms of these methods are apparently due to misunderstandings of the nature and theoretical principles behind this technique. The random matrix approach is NOT based on the principle of "minimum information gain," and the random matrix approach does NOT assume that coefficients in inputoutput matrices are really random numbers without any recognizable economic relationships between coefficients.

The "random matrix" approach assumes knowledge of only a minimal subset of the data in a complete input-output matrix and generates multiplier estimates subject to this restriction. Thus, the "random matrix" approach can provide estimators based on all possible scenarios of information content about the input-output matrix. These shortcut multiplier estimators are the means of the distribution of multipliers induced over all possible input-output matrices subject to the known information constraints. The effectiveness and reliability of these shortcut formulas is directly inferred from the size of the standard error (standard deviation) of the estimated multiplier distribution. This standard deviation decreases as the known information content of the input-output matrix increases.

For example, suppose that no data are known about the input-output matrix. Then the "random matrix" approach yields output multiplier estimates with some unknown mean and infinite standard errors. Thus, no information about the coefficient matrix yields no information about the output multipliers but at zero cost. At the opposite extreme, suppose that the complete input-output matrix is known with certainty. Then, the output multiplier estimates are determined completely and the standard error of the distribution of multiplier estimates is 0 . Therefore, complete knowledge of the coefficient matrix provided exact output multiplier values but at a very high cost. Partial knowledge about the input-output matrix results in a standard deviation of the induced multiplier distribution between 0 and infinity. The effectiveness of a particular shortcut multiplier formula will be contingent on the tradeoff between the level of information about the input-output matrix required and the size of the standard deviation of the multiplier distribution. If this standard deviation is small, then there is a high probability that the actual output multiplier is very close to the shortcut formula, which is the mean of the distribution of multipliers. The "random matrix" approach produces a formula that is a function of the known information from the input-output coefficient matrix. Logically, the more industry detail that is available from the inputoutput coefficient matrix, the more complex and accurate the multiplier formula, although the higher the data collection cost in utilizing the shortcut formula. Ultimately, one desires a shortcut multiplier formula that requires a minimum amount of industry detail from the input-output coefficient matrix but still provides close and acceptably accurate estimates of the impact with small errors.

The shortcut output multiplier formula in equation (1) was derived by direct application of the "random matrix" approach to the case where only the column totals of the coefficient matrix were assumed to be known. Let $\mathrm{R}$ represent the set of all nonnegative matrices with column totals equal to the known fixed values. The actual but unknown input-output matrix must be contained in the set $\mathbf{R}$. Furthermore, each matrix in $R$ has an equal probability of being the true matrix because all the known information about the 
input-output matrices has already been incorporated in our restriction to matrices in the set $\mathbf{R}$, and therefore, there is no way to make further probability distinctions between them. Equal probability weights for each matrix in $\mathbf{R}$ allows the coefficients over the matrices in $\mathbf{R}$ to be random variables. Furthermore, since no further prior information about the matrices in $\mathrm{R}$ is known other than the fixed column totals, then coefficient random variables in the same column must be identically distributed as there is no way to distinguish between the probability distributions for random variable coefficients in the same column.

Harrigan (1982) criticizes this "random matrix" approach on two counts. First, he observes that much is known about the structure of the input-output matrix which is inconsistent with the idea of identically distributed coefficient random variables in the same column. Secondly, Harrigan suggests that information about the location of zero cells and large coefficients and other structural relationships in the economy could be incorporated into the analysis to improve the multiplier estimates. He supports his claim by providing an empirical example.

While Harrigan's second observation is certainly true, it belies the main thrust of this research which is to discover shortcut formulas with minimal amounts of data that still produce acceptable output multiplier estimates. One can always argue that more information, more structure, larger and more complex models, and an increased number of endogenous variables, will theoretically produce better results. Yes, but at a much higher cost in time and monetary expense.

Harrigan questions whether the "random matrix" approach can provide acceptable estimates of the output multipliers as functions of the column totals because the coefficient random variables in the same column become identically distributed. It must be emphasized, however, that this fact does not imply that coefficients in a given column of any input-output matrix are actually randomly distributed or that they are actually asssumed to be equal. Rather, identically distributed coefficients in the same column are due to the fact that, in our experiment, only the column totals of the inputoutput matrix are given and no other specific information about the individual coefficients is known. Harrigan's query is critical as it addresses the main issue as to what are the key factors in the input-output matrix that form the size of the output multipliers. If the coefficients individually play a major role, then shortcut formulas based solely on the column totals are doomed to $\mathrm{xe}$ wildly erratic estimators.

The standard deviation of the output multiplier distribution, as well as the minimum and maximum possible values a multiplier could have, are measures of the effectiveness of the shortcut formula. If the standard deviation of the output multiplier distribution for a sector $\mathrm{j}$ is relatively small, then one can conclude, based on statistical theory, that knowledge of only the column totals of the input-output matrix produces output multipliers for industry $j$ that fall, with a high probability, in a very small interval around the point estimate given in equation (1). However, if the standard deviation of the output multiplier distribution for a sector $\mathrm{j}$ is relatively large, then knowledge of only the column totals of the input-output matrix would produce output multipliers for industry $\mathrm{j}$ that fall, with a high probability, in a very large interval around the point estimate given in equation (1), which implies that knowledge of the column totals alone is not sufficient to produce reliable output multiplier estimates. Fortunately for economic impact analysis, the standard deviation tends to be very small. Knowledge of only the column totals of the coefficient matrix produces very small standard errors for the distribution of output multipliers, which implies that the mean of the output multiplier distribution has a high probability of being close to the output multiplier that would be found in a complete input-output analysis. In Burford and Katz (1977), the random matrix experiment was simulated for several input-output models and the standard deviations of the output multipliers were found to be very small. Furthermore, a close approximation of the variance of the output multiplier distribution (Burford and Katz (1977)) has been derived and verified by simulation. Thus, we conclude that the column totals are the key determinants of the output multipliers, although the individual coefficients play "some" role and can have a modest impact on the size of the output multiplier. However, it rarely occurs that individual coefficients have more than a minor effect on the actual multipliers, and when this does occur, it is usually in small highly aggregaged models and is largely a function of how sectors are aggregated. Although the individual coefficients are important in representing the economic structure of the economy through the input-output matrix, they play a relatively minor role in the formation of the output multiplier.

Harrigan (1982) suggests an alternative to the random matrix approach that incorporates addtional information concerning earlier input-output tables for the region (which may not exist) into the estimation process. Furthermore, Katz and Burford (1981) have derived additional shortcut multiplier formulas assuming additional information from the coefficient matrix. However, it is not our position that one particular shortcut output multiplier, or an input-output model at a particular level of disaggregation embodies the "best" 
results from weighting each matrix in $R$ equally. Let the Leontief inverse matrix be represented by the matrix $Q=(I-A)^{-1}$ with associated random variables $q_{i j}^{\text {ss }}$ of $Q$. Furthermore, if

$\sum_{r=1}^{2} \sum_{i=1}^{n} \operatorname{Cov}\left(a_{k i}^{s r}, q_{i h}^{r s}\right) \tilde{=} 0$,

$$
1 \leq \mathrm{h} \leq \mathrm{n}, 1 \leq \mathrm{k} \leq \mathrm{n}, \mathrm{s}=1,2 \text {, }
$$

then the expected impact of the column of Leontief inverse coefficients for industry $h$ in region $r, s$ represents the other region, is

$$
E\left(q_{h h}^{r r}\right)=1+\frac{\left(w_{h}^{\pi r} / n\right)\left(1-\bar{w}^{s s}\right)+\left(w_{h}^{s r} / n\right)\left(w^{r s}\right)}{\left(1-\bar{w}^{m}\right)\left(1-\bar{w}^{s s}\right)-\left(\bar{w}^{r s} \bar{w}^{-s r}\right)}
$$

$E\left(q_{k j}^{\pi}\right)=E\left(q_{h h}^{\pi}\right)-1 \quad k \neq j$.

$$
E\left(q_{k j}^{s r}\right)=\frac{\left(w_{h}^{s r} / n\right)\left(1-\bar{w}^{r r}\right)+\left(w_{h}^{r r} / n\right)\left(w^{s r}\right)}{\left(1-\bar{w}^{\text {Ir }}\right)\left(1-\bar{w}^{s s}\right)-\left(\bar{w}^{\mathrm{rs}} \bar{w}^{\mathrm{sr}}\right)}
$$

Proof: The expected values of the inverse coefficients will be found by solving a system of equations that relates the input-output and inverse coefficient random variables. Since the Leontief inverse is

it follows that

$$
Q=(I-A)^{-1} \text {, }
$$

$$
\text { (I - A) } \mathrm{Q}=\mathrm{I} \text {. }
$$

For a two region model, equation (4) represents $4 n^{\prime \prime}$ separate subequations that follow when setting corresponding matrix coefficients on both sides equal. Assume without loss of generality that $h$ is a sector in region 1. Equating the 2 n coefficients in column $h$ of region 1 on both sides of equation (4), yields the following system of equations of random variables.

$$
\begin{aligned}
& q_{1 h}^{11}=\sum_{r=1}^{2} \sum_{i=1}^{n} a_{1 i}^{1 r} q_{i h}^{r 1} \cdots \\
& q_{n h}^{11}=\sum_{r=1}^{2} \sum_{i=1}^{n} a_{n i}^{1 r} q_{i h}^{r l} \\
& q_{h h}^{11}=1+\sum_{r-1}^{2} \sum_{i=1}^{n} a_{h i}^{1 r} q_{i h}^{r l} \cdots
\end{aligned}
$$

$$
\begin{aligned}
q_{1 h}^{21} & =\sum_{r=1}^{2} \sum_{i=1}^{n} a_{1 i}^{2 r} q_{i h}^{r 1} \cdots \\
q_{n h}^{21} & =\sum_{r=1}^{2} \sum_{i=1}^{n} a_{n i}^{2 r} q_{i h}^{r l}
\end{aligned}
$$

Taking the expected values of the $2 n$ random variable equations in (5) yields

$$
\begin{gathered}
E\left(q_{1 h}^{11}\right)=\sum_{r=1}^{2} \sum_{i=1}^{n} E\left(a_{1 i}^{1 r} E\left(q_{i h}^{r 1}\right)\right. \\
+\sum_{r=1}^{2} \sum_{i=1}^{n} \operatorname{Cov}\left(a_{1 i}^{1 r}, q_{i h}^{r 1}\right) \ldots . \\
E\left(q_{h h}^{11}\right)=1+\sum_{r=1}^{2} \sum_{i=1}^{n} E\left(a_{h i}^{1 r}\right) E\left(q_{i h}^{r 1}\right) \\
\quad+\sum_{r=1}^{2} \sum_{i=1}^{n} \operatorname{Cov}\left(a_{h i}^{1 r}, q_{i h}^{r 1}\right) \ldots . \\
E\left(q_{n h}^{11}\right)=\sum_{r=1}^{2} \sum_{i=1}^{n} E\left(a_{n i}^{1 r}\right) E\left(q_{i h}^{r 1}\right) \\
+\sum_{r=1}^{2} \sum_{i=1}^{n} \operatorname{Cov}\left(a_{n i}^{1 r}, q_{i h}^{r 1}\right) \\
E\left(q_{1 h}^{21}\right)=\sum_{r=1}^{2} \sum_{i=1}^{n} E\left(a_{1 i}^{2 r}\right) E\left(q_{i h}^{r 1}\right) \\
\quad+\sum_{r=1}^{2} \sum_{i=1}^{n} \operatorname{Cov}_{\left(a_{1 i}^{2 r}, q_{i h}^{r 1}\right)}^{r 1} \cdot . \\
E\left(q_{n h}^{21}\right)=\sum_{r=1}^{2} \sum_{i=1}^{n} E\left(a_{n i}^{2 r}\right) E\left(q_{i h}^{r 1}\right) \\
+\sum_{r=1}^{2} \sum_{i=1}^{n} \operatorname{Cov}\left(a_{n i}^{2 r}, q_{i h}^{r 1}\right)
\end{gathered}
$$

Since each matrix in $\mathbf{R}$ is equally probable and the only constraints on the coefficient random variables are their fixed column totals in the interregional submatrix, then coefficient random variables in different industry columns of $\mathbf{A}$ matrix and different interregional submatrices are independent whereas coefficients in the same column and interregional matrix are dependent and identically distributed. Therefore, the expected value or mean of each coefficient random variables $a_{i j}$ can be derived as follows (also see Katz and Burford (1981)). For a given column $\mathrm{j}$ in the rxs regional submatrix, the sum of the rapdom variables in that column is fixed to the known $w_{j}$. Thus,

$$
a_{1 j}^{r s}+\ldots+a_{n j}^{r s}=w_{j}^{r s}
$$

Taking the expected value of both sides of the random variable equation gives:

$$
E\left(a_{i j}^{r s}\right)+\ldots+E\left(a_{n j}^{r s}\right)=E\left(w_{j}^{r s}\right)=w_{j}^{r s}
$$


or "most preferable" method of measuring economic impacts. On the contrary, both shortcut formulas and input-output models provide a wide range of techniques with varying levels of accuracy, cost and impact detail for the analyst to select from. Additional comments defending the shortcut multiplier approach can be found in Katz and Burford (1982) and Burford and Katz (1985).

\section{The Development of Shortcut Interregional Multipliers}

Consider a 2 region interregional model with $n$ industry sectors per region and define " $A$ " to represent the interregional input-output coefficient matrix for this model partitioned as

$$
A=\left[\begin{array}{ll}
A^{11} & A^{12} \\
A^{21} & A^{22}
\end{array}\right]
$$

where $A^{11}$ and $A^{22}$ are the input-output coefficient matrices for region 1 and 2 respectively, while $A^{21}$ and $A$ are the matrices of the proportion of interregional purchase coefficients. The coefficients of the partitioned matrix $\mathrm{A}^{\mathrm{rs}}, \mathrm{r}=1,2, \mathrm{~s}=1,2$ is notationally represented by

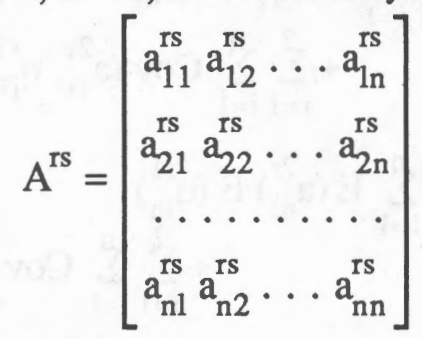

Define $w_{h}{ }^{r s}$ to be the column total of the coefficient matrix of industry $h_{h}$ in region s spent on region $r$, or equivalently, the proportion of total expenditures of industry $h$ in region $s$ on region $r$. The shortcut formulas that are derived here assume knowledge of all the column totals of the regional $\left(\mathrm{A}^{11}, \mathrm{~A}^{22}\right)$ and interregional matrices $\left(A^{12}, A^{21}\right)$. Again, our primary motive in requiring knowledge of this data is to determine the minimal amount of information needed about the interregional matrix to assure accurate estimates of the multipliers. Although shortcut formulas can theoretically be derived for scenarios which involve more or less data from the interregional matrix, these changes would impact either the accuracy of the multipliers or the cost of producing the estimates. For example, suppose that only the gross column totals of the interregional matrix were assumed kown. Although estimates of the various regional, interregional, and total output multipliers could be derived, only the total output multiplier impact over all regions would tend to be accurately estimated as the "random matrix" approach would yield regional and interregional output multiplier impact distributions with large standard deviations which would detract from the usefulness of these estimates.

Define $R$ to be the set of all possible nonnegative $2 n \times 2 n$ matrices which satisfy the known column total constraints for both the regional and interregional matrices for a two region model. Then, the actual interregional input-output matrix is theoretically contained in the set $R$, but it cannot be distinguished from others since only the column totals of the regional and interregional matrices are presumed known. Thus, weight each matrix in $\mathrm{R}$ with equal probability, which induces a probability distribution on the coefficients over the set of matrices in $R$.

Let $Q=(I-A)^{-1}$ be the Leontief inverse matrix and partition $\mathrm{Q}$ as

$$
\mathrm{Q}=\left[\begin{array}{ll}
\mathrm{Q}_{21}^{11} & \mathrm{Q}_{22}^{12} \\
\mathrm{Q}^{12} & \mathrm{Q}^{2}
\end{array}\right]
$$

where $Q^{r s}$ represents the matrix of direct and indirect impacts of region $s$ on region $r$, and define each partitioned matrix $Q^{\mathrm{rs}}$ to have coefficients denoted as



Since the Leontief inverse matrix can be computed for each matrix in the set $\mathbf{R}$, the probability distribution on the coefficients over the matrices in $R$ induces a probability distribution on the coefficients of the Leontief inverse matrix and multipliers that correspond to each matrix in $\mathbf{R}$. The shortcut formulas that are derived here approximate the mean or expected value of the distribution of Leontief inverse coefficients and multipliers and are functions of the known column totals.

\section{Derivation of Shortcut Formulas}

Theorem: Let $\mathrm{R}$ be the set of all possible two region $2 n \times 2 n$ interregional input-output matrices with fixed regional and interregional column totals

$\mathrm{w}_{\mathrm{h}}^{\mathrm{rs}}, \mathrm{h}=1,2, \ldots \mathrm{n}, \mathrm{r}=1,2, \mathrm{~s}=1,2$. Define $\overline{\mathrm{w}}^{\mathrm{rs}}$ to be the average over all column totals $w_{h}^{\text {rs }}$ in the rxs interregional submatrix $\left(A^{\mathrm{rs}}\right)$. Let $a_{i j}^{\text {ts }}$ represent the random variable of the $i, j$ position in the rxs interregional submatrix over all the matrices in $\mathrm{R}$ that 
Since coefficient random variables in the same column and regional submatrix are identically distributed, therefore,

$$
\begin{aligned}
n E\left(a_{i j}^{r s}\right. & =w_{j}^{r s} \\
E\left(a_{i j}^{r s}\right. & =w_{j}^{r s} / n .
\end{aligned}
$$

The system of equations in (6) will be reduced further by dropping the sums of the covariance terms. If these covariance sums are all approximately zero, then dropping these terms will have a negligible effect on the resulting multiplier estimates. It must be emphasized that the covariance relationship between a coefficient random variable in A and its corresponding inverse term that is expressed in the covariance sums is a direct product of the assumption that each matrix in $\mathbf{R}$ is equally likely. Thus, the covariance terms have no true economic interpretation with respect to the underlying structural relationship between individual industry sectors. Katz and Burford (1981) have deductively reasoned that similar kinds of covariance terms were approximately zero in the application of the random matrix approach to regional models. Furthermore, as in most cases when an analytical solution is extremely difficult or impossible to derive, lengthy simulations which directly measured these covariance sums, have found them to be negligible. The next section provided additional confirmation of the negligible effect of the covariance sums as part of the empirical testing of the interregional shortcut multiplier formulae.

Applying the expected value derivations and covariance assumptions to the previous system of equations in (6) yields

$$
\begin{aligned}
& E\left(q_{1 h}^{11}\right)=\sum_{r=1}^{2} \sum_{i=1}^{n}\left(w_{i}^{1 r} / n\right) E\left(q_{i h}^{r 1}\right) \cdots \\
& E\left(q_{h h}^{11}\right)=1+\sum_{r=1}^{2} \sum_{i=1}^{n}\left(w_{i}^{1 r} / n\right) E\left(q_{i h}^{r 1}\right) \cdots \\
& E\left(q_{n h}^{11}\right)=\sum_{r=1}^{2} \sum_{i=1}^{n}\left(w_{i}^{1 r} / n\right) E\left(q_{i h}^{r l}\right) \\
& E\left(q_{l h}^{21}\right)=\sum_{r=1}^{2} \sum_{i=1}^{n}\left(w_{i}^{2 r} / n\right) E\left(q_{i h}^{r l}\right) \ldots \\
& E\left(q_{n h}^{21}\right)=\sum_{r=1}^{2} \sum_{i=1}^{n}\left(w_{i}^{2 r} / n\right) E\left(q_{i h}^{r 1}\right)
\end{aligned}
$$

The system of equations in (10) represents $2 n$ simultaneous equations in the expected values $E\left(q_{1 h}^{11}\right), E\left(q_{2 h}^{11}\right), \ldots, E\left(q_{n h}^{11}\right), E\left(q_{1 h}^{21}\right), E\left(q_{2 h}^{21}\right)$, ..., $E\left(q_{n h}^{21}\right)$. Since the right hand side sums are identical, it follows that

$$
\begin{aligned}
E\left(q_{1 h}^{11}\right) & =E\left(q_{j h}^{11}\right), \\
j & =2,3, \ldots h-1, h+1, \ldots n, \\
E\left(q_{1 h}^{21}\right) & =E\left(q_{j h}^{21}\right), \quad j=2,3, \ldots n
\end{aligned}
$$

and $E\left(q_{1 h}^{11}\right)=E\left(q_{h h}^{11}\right)-1$

Let $X=E\left(q_{1 h}^{11}\right)$ and $Y=E\left(q_{1 h}^{21}\right)$. Then the following two equations from the system in (10)

$$
\begin{aligned}
& E\left(q_{1 h}^{11}\right)=\sum_{r=1}^{2} \sum_{i=1}^{n}\left(w_{i}^{1 r} / n\right) E\left(q_{i h}^{r 1}\right) \\
& E\left(q_{1 h}^{21}\right)=\sum_{r=1}^{2} \sum_{i=1}^{n}\left(w_{i}^{2 r} / n\right) E\left(q_{i h}^{r 1}\right)
\end{aligned}
$$

can be rewritten as,

$$
\begin{aligned}
& X=\left(w_{h}^{11} / n\right)+\sum_{i=1}^{n}\left(w_{i}^{11} / n\right)(X)+\sum_{i=1}^{n}\left(w_{i}^{12} / n\right)(Y) \\
& Y=\left(w_{h}^{21} / n\right)+\sum_{i=1}^{n}\left(w_{i}^{21} / n\right)(X)+\sum_{i=1}^{n}\left(w_{i}^{22} / n\right)(Y) \\
& \text { or } X=w_{h}^{11} / n+\left(w^{11}\right)(X)+\left(w^{12}\right)(Y) \\
& \text { and } Y=w_{h}^{21} / n+\left(w^{21}\right)(X)+\left(w^{22}\right)(Y)
\end{aligned}
$$

This system of two linear equations in the unknowns $\mathbf{X}$ and $\mathrm{Y}$ can be solved by Cramer's rule yielding the solutions presented in (2). Q.E.D.

The shortcut formulas are the means or expected values of the distributions of multipliers that are induced by the set of matrices in R. By definition, the output impact of industry $h$ in region $r$ on its own region is

$$
u_{h}^{\pi}=\sum_{i=1}^{n} q_{i h}^{\pi}
$$

Therefore, the shortcut output multiplier estimate of $u_{h}{ }_{h}$ is

$$
\begin{aligned}
E\left(u_{h}^{\mathrm{rr}}\right) & =\sum_{i=1}^{\mathrm{n}} \mathrm{E}\left(\mathrm{q}_{\mathrm{ih}}^{\mathrm{rr}}\right) \\
& =1+\frac{\left(\mathrm{w}_{\mathrm{h}}^{\mathrm{rr}}\right)\left(1-\overline{\mathrm{w}}^{\mathrm{ss}}\right)+\left(\mathrm{w}_{\mathrm{h}}^{\mathrm{sr}}\right)\left(\overline{\mathrm{w}}^{\mathrm{rs}}\right)}{\left(1-\overline{\mathrm{w}}^{\mathrm{rr}}\right)\left(1-\overline{\mathrm{w}}^{\mathrm{ss}}\right)-\overline{\mathrm{w}}^{\mathrm{rs}} \overline{\mathrm{w}}^{\mathrm{sr}}},
\end{aligned}
$$


where $s$ represents the other region. Furthermore, the impact on the other region $\mathrm{s}$ by industry $\mathrm{h}$ in region $r$ is defined to be

$$
u_{h}^{s r}=\sum_{i=1}^{n} q_{i h}^{s r},
$$

and the shortcut formula estimate of this impact is

$$
\begin{aligned}
E\left(u_{h}^{s r}\right) & =\sum_{i=1}^{n} E\left(q_{i h}^{s r}\right), \\
& =\frac{\left(w_{h}^{s r}\right)\left(1-\bar{w}^{\pi r}\right)+\left(w_{h}^{\text {Ir }}\right)\left(\bar{w}^{s r}\right) .}{\left(1-\bar{w}^{-I r}\right)\left(1-\bar{w}^{s s}\right)-\bar{w}^{-{ }^{T S}} \bar{w}^{s r}}
\end{aligned}
$$

Finally, the total ouptut impact of industry $h$ in region $r$ on both regions is defined to be

$$
u_{h}^{\cdot r}=u_{h}^{1 r}+u_{h}^{2 r} \text {, }
$$

whereas the shortcut formula is

$$
E\left(u_{h}^{\cdot r}\right)=E\left(u_{h}^{1 r}\right)+E\left(u_{h}^{2 r}\right) .
$$

Let $b_{h .}^{T}$ represents the proportion of household payments by industry $h$ in region $r$. Then the direct and indirect income multiplier effect of industry $h$ in region $r$ on region $s$ is defined to be

$$
r_{h}^{s r}=\sum_{i=1}^{n} b_{i}^{r} q_{i h}^{s r},
$$

and that the total direct and indirect income multiplier effect of industry $h$ in region $r$ on both regions is defined to be

$$
\mathrm{I}_{\mathrm{h}}^{\mathrm{r}}=\mathrm{I}_{\mathrm{h}}^{1 \mathbf{r}}+\mathrm{I}_{\mathrm{h}}^{2 \mathrm{r}}
$$

Let $5^{\mathrm{r}}$ represent the average household coefficient over all industries in region $r, r=1,2$. Then the shortcut direct and indirect income multiplier formula is

$$
\begin{aligned}
E\left(I_{h}^{s r}\right) & =\sum_{i=1}^{n} E\left(b_{i}^{r} q_{i h}^{s r}\right) \\
& = \begin{cases}5^{s} E\left(u_{h}^{s r}\right) & \text { if } r \neq s \\
5^{r}\left(E\left(u_{h}^{r r}\right)-1\right)+b_{h}^{r} & \text { if } r=s .\end{cases}
\end{aligned}
$$

Also, the shortcut formula for the total income multiplier $\mathrm{I}_{\mathrm{h}}^{\mathrm{r}}$ is

$$
E\left(I_{h}^{\cdot r}\right)=E\left(I_{h}^{1 r}\right)+E\left(I_{h}^{2 r}\right) .
$$

Finally, let $e_{h}^{r}$ represents the empioyment per output of industry $h$ in region $r$. Then the direct and indirect employment multiplier effect of industry $h$ in region $r$ on region $S, Z_{h}^{s r}$, is defined to be

$$
\mathrm{Z}_{\mathrm{h}}^{\mathrm{sr}}=\sum_{\mathrm{i}=1}^{\mathrm{n}} \mathrm{e}_{\mathrm{i}}^{\mathrm{r}} \mathrm{q}_{\mathrm{ih}}^{\mathrm{sr}}
$$

and the total direct and indirect employment multiplier effect of industry $h$ in region $r$ on all regions, $\dot{Z}_{h}$, is

$$
\dot{\mathrm{Z}}_{\mathrm{h}}^{\mathrm{r}}=\mathrm{E}\left(\mathrm{Z}_{\mathrm{h}}^{1 \mathrm{r}}\right)+\mathrm{E}\left(\mathrm{Z}_{\mathrm{h}}^{2 \mathrm{r}}\right)
$$

Let $\mathrm{e}^{\mathrm{T}}$ represent the average employment per output over all industries in region $r, r=1,2$. In a similar fashion to the derivation of the shortcut direct and indirect income multipliers, the shortcut direct and indirect employment multiplier impacts of industry $h$ in region $\mathrm{r}$ on another region $\mathrm{s}$ can be determined to be

$E\left(Z_{h}^{s r}\right)= \begin{cases}e^{-s} E\left(u_{h}^{s r}\right) & \text { if } r \neq s \\ e^{-r}\left(E\left(u_{h}^{r r}\right)-1\right)+e_{h}^{r} & \text { if } r=s\end{cases}$

and the shortcut formula of the total employment multiplier impact of industry $h$ in region $r$ over both regions is

$$
E\left(Z_{h}^{r}\right)=E\left(Z_{h}^{1 r}\right)+E\left(Z_{h}^{2 r}\right) .
$$

These shortcut formulae can also produce estimates of the interregional feedback effects. Consider a two region model where industry $h$ is in region $r$ and $\mathrm{S}$ represents the other region. The regional output multiplier effect of industry $h$ only in region $r$ is given by the following formula, stated earlier as Equation (1), $1+\left(w_{h}^{\mathrm{Tr}} /\left(1-\bar{w}^{\mathrm{II}}\right)\right)$. Therefore, the inters gional output feedback effect of industry in region is

Output Feedback

$$
\begin{aligned}
& =E\left(u_{h}^{\mathrm{Ir}}\right)-\left(1+\left(\mathrm{w}_{\mathrm{h}}^{\mathrm{rr}} /\left(1-\overline{\mathrm{w}}^{\mathrm{rr}}\right)\right)\right) \\
& =\frac{\left(1-\overline{\mathrm{w}}^{\mathrm{m}}\right)\left(\mathrm{w}_{\mathrm{h}}^{\mathrm{sr}} \overline{\mathrm{w}}^{\mathrm{rs}}\right)+\mathrm{w}_{\mathrm{h}}^{\mathrm{r}} \overline{\mathrm{w}}^{\mathrm{rs}} \overline{\mathrm{w}}^{\mathrm{sr}}}{\left(1-\overline{\mathrm{w}}^{\mathrm{rr}}\right)^{2}\left(1-\overline{\mathrm{w}}^{\mathrm{ss}}\right)-\left(1-\overline{\mathrm{w}}^{\mathrm{r}}\right) \overline{\mathrm{w}}^{\mathrm{rs}} \overline{\mathrm{w}}^{\mathrm{sr}}}
\end{aligned}
$$

The following conclusions follow immediately from equation (25);

(1) If there is no economic linkage between regions rand $\mathrm{S}$, then both $\frac{\mathrm{w}}{\mathrm{W}}$ and $\frac{\mathrm{sr}}{\mathrm{W}}$ are zero and there are no feedback effects.

(2) The larger the direct proportion spent by industry $h$ in region $r$ on region $s$, the larger the value for $w_{h}{ }_{h}$ and the higher the feedback effect.

(3) The greater the linkage between the and $\mathrm{S}$ regions, the greater the values of $\overrightarrow{\mathrm{W}}$ and $\overrightarrow{\mathrm{W}}$ T and the larger the feedback effects.

These conclusions are consistent with earlier evaluations of the feedback effects as noted by Miller (1969). 
Theorem 1 can be generalized to provide estimates of the Leontief inverse for $\mathrm{k}$ regions with $\mathrm{n}$ industry sectors per region. The column of inverse coefficients for industry $h$ in region $r$, are estimated by (det $=$ determinant)

$$
\begin{aligned}
& E\left(q_{i h}^{\mathrm{II}}\right)=\frac{\operatorname{det}\left(\left(I-W^{\mathrm{r}}\right)\right.}{\operatorname{det}(I-W)} \\
& E\left(q_{h h}^{\mathrm{Ir}}\right)=1+\frac{\operatorname{det}\left(\left(I-W^{\mathrm{r}}\right)\right.}{\operatorname{det}(I-W)} \\
& E\left(q_{i h}^{\mathrm{sr}}\right)=\frac{\operatorname{det}\left(\left(I-W^{s}\right)\right.}{\operatorname{det}(I-W)}
\end{aligned}
$$$$
\text { for ifh }
$$

for $\mathrm{r} \neq \mathrm{s}$

whereWis thekxkmatrix of average submatrix column totals over all $\mathrm{k}$ regions



and $\left(I-W^{r}\right)$ is the matrix $(I-W)$ with the rth column replaced by the elements $w_{h} / n, w_{h}^{2 r} / n, \ldots, w_{h}^{k r} / n$. These expected value estimates of the inverse coefficients, utilized in a similar fashion in the derivation of the formulas for the two region case, generate interregional shortcut multiplier formula estimates for output, income and employment.

\section{Empirical Tests}

This section presents empirical evidence to support the validity and accuracy of the interregional shortcut multiplier formulas that were developed in the previous section. The purpose of these experiments is to answer the following two questions. (1) Do the shortcut multiplier formulas adequately approximate the corresponding multipliers from a complete interregional model? (2) Do the covariance assumptions that were made in the derivation of the mean of the multiplier distributions significantly distort the shortcut multiplier formulas? The first experiment is performed using the data from the interregional input-output model by West,
Morrison, and Jensen (1982) for the two regions Queensland and Victoria which contains three industries per region. Five hundred input-output matrices were randomly generated from the set of matrices where the industry submatrix column totals were fixed with the exact values reported by West, et al. The output and income multipliers were computed for each of the 500 matrices and summary statistics were computed for both types of multipliers. Table 1 presents the results of these experiments.

The first column labelled (1) indicates the impacted region along with the specific industry whose impact is being measured. Industries $1,2,3$, are from region 1 (Queensland) while industries 4, 5, 6, are from region 2 (Victoria). Column (2) presents the average multiplier over the 500 random matrices that were generated by the simulation, whereas column (3) displays the shortcut formula estimate of the average or expected value of the multiplier. Since the simulated multipliers in column (2) were generated without any assumptions about covariances, the fact that columns (2) and (3) tend to be extremely close supports the derived shortcut multiplier formula as the mean or expected value over all the matrices in the set of random matrices despite the assumption of negligible covariance sums. Column (4) indicates the actual multiplier effect that was found by West, et al. using the full interregional model. Column (5) displays the standard deviation of the 500 simulated multipliers, while columns (6) and (7) reflect the minimum and maximum multiplier values over the set of the 500 randomly generated matrices. The general small value for the standard deviation of the multipliers is an indication that the multiplier values in the set of random matrices do not vary a great deal from the mean, which makes the mean a good estimate of the actual multiplier impact. Furthermore, the range from the minimum to the maximum multiplier is in most cases approximately six standard deviations and the actual multiplier generated by West, et al. always fell within these minimum and maximum multiplier limits.

A second experiment was run to evaluate the performance of the shortcut multiplier formulas on larger regions by examining the three region interregional model for the Netherlands by Oosterhaven (1981). The model subdivides the Netherlands into three separate regions; Northern Netherlands (region 1), Rijnmond (region 2) and the rest of the Netherlands (region 3). Each region contains 23 industry sectors.

Table 2 reveals summary measures of the effectiveness of the shortcut formulas in approximating the actual output and direct and indirect income multipliers for the Dutch model. For the regional 
Table 1

A Comparison of Shortcut Multiplier Formulas for the Queensland-Victoria Interregional Model

Output Multiplier

(1)

Region and

Industry

$1 \quad 1$

12

13

14

15

16

21

22

23

24

25

26

TOTAL 1

TOTAL 2

TOTAL 3

TOTAL 4

TOTAL 5

TOTAL 6
(2)

Simulation
Mean

1.5049

1.7584

1.3499

.0018

.0026

.0012

.0737

.1102

.0669

1.1775

1.5026

1.2013

\subsection{5}

1.8686

1.4168

1.1793

1.5052

1.2025
(3)

Formula
Mean

1.5030

1.7546

1.3498

.0018

.0026

.0012

.0734

.1094

.0669

1.1767

1.4994

1.2017

1.5765

1.8641

1.4167

1.1784

1.5020

1.2029
(4)

Actual

Multiplier

1.5089

1.7718

1.3453

.0019

.0030

.0013

.0848

.1226

.0745

1.1910

1.5722

1.2195

1.5937

1.8944

1.4198

1.1929

1.5802

1.2208
(5)

Standard
Deviation

.0315

.0560

.0227

.0001

.0002

.0001

.0051

.0083

.0039

.0111

.0357

.0124

.0365

.0643

.0266

.0112

.0359

.0125
(6)

Minimum

Multiplier

1.4412

1.6566

1.2960

.0016

.0021

.0010

.0624

.0946

.0576

1.1593

1.4536

1.1822

1.5036

1.7512

1.3536

1.1608

1.4557

1.1833
(7)

Maximum

Multiplier

1.6055

1.9504

1.4279

.0022

.0033

.0014

.0913

.1357

.0807

1.2149

1.6227

1.2402

1.6969

2.0861

1.5086

1.2171

1.6260

1.2417 submatrix indicated in column (1), column (2) displays the average of the actual total output effect on the row region over all 23 sectors in the column region. For example, the average output effect by the 23 sectors in the Northern Netherlands on Rijnmond (column region $=1$, row region $=2$ ) is .0315 . Column (3) specifies the mean absolute difference for each regional submatrix between the actual output effects and the estimated output effects by the appropriate shortcut multiplier. Column (4) presents the mean absolute percent difference between the actual and estimated output effects from the shortcut formula. The direct and indirect income multiplier portion of the table is defined in a similar manner.

Overall, the small mean absolute differences between the actual and estimated multiplier effects for each subregional matrix establishes the usefulness of the shortcut multiplier formula as an effective estimator of the reported multipliers. Although the mean absolute percent error between the actual and estimated multiplier for a subregional matrix is also shown in Table 2, it is not a reliable measure of the effectiveness of the shortcut formulas in cases where the average multiplier impact is small. Although multipliers for input-output tables have historically been reported to four of five decimal places, it is senseless to expect accuracy at more than one or two decimal places, even in the best studies, due to possible sampling and data collection problems.

To test the acceptability of the covariance assumptions, five hundred input-output matrices were randomly generated from the set of matrices where the 
Table 1 (Continued)

Direct and Indirect Income Multiplier

(1)

\section{Region and Industry}

\section{1}

12

13

14

15

16

$\begin{array}{ll}2 & 1 \\ 2 & 2 \\ 2 & 3 \\ 2 & 4 \\ 2 & 5 \\ 2 & 6\end{array}$

$\begin{array}{ll}\text { TOTAL } & 1 \\ \text { TOTAL } & 2 \\ \text { TOTAL } & 3 \\ \text { TOTAL } & 4 \\ \text { TOTAL } & 5 \\ \text { TOTAL } & 6\end{array}$
(2)

Simulation
Mean

\section{.2298}

.4264

.5012

.0005

.0006

.0003

.0221

.0328

.0200

.1994

.4368

.5247

.2519

.4592

.5211

.1999

.4374

.5250
(3)

Formula
Mean

.2317

.4237

.5003

.0004

.0006

.0003

.0219

.0327

.0200

.2002

.4330

.5246

.2536

.4564

.5203

.2007

.4337

.5249
(4)

Actual Multiplier

\section{.243}

.423

.522

.001

.001

.000

.026

.037

.024

.203

.449

.540

.269

.460

.546

.204

.450

.540
(5)

Standard

Deviation

.0245

.0337

.0146

.0001

.0001

.0001

.0032

.0052

.0031

.0100

.0296

.0120

.0277

.0389

.0177

.0101

.0297

.0120
(6)

(7)

Minimum

Multiplier

Maximum

Multiplier

.1594

.3303

.4630

.0002

.0004

.0002

.0131

.0194

.0118

.1729

.3631

.4943

.1724

.3497

.4748

.1731

.3635

.4945
.2740

.4929

.5336

.0006

.0009

.0004

.0307

.0529

.0280

.2188

.4874

.5492

.3047

.5459

.5616

.2194

.4843

.5496 industry submatrix column totals were fixed with the exact values reported by Oosterhaven (1981). The output and direct and indirect income multipliers were computed for each of the 500 matrices broken down over all nine regional submatrices. For each sector, the simulation mean of the 500 multipliers, which is generated without recourse to any theoretical assumptions, and the formula mean multiplier, which was derived in Theorem 1 and is subject to covariance assumptions, both approximate the true mean of the multiplier distribution. Results of the simulation tests on the three region interregional model for the Netherlands are displayed in Table 3.
For each regional submatrix indicated in column (1), column (2) again indicates the average of the actual total output effects on the row region over all 23 sectors in the column region. Column (3) specifies the mean absolute difference for each regional submatrix between the simulation mean of the multiplier effect and the corresponding shortcut multiplier formula estimate, and column (4) provides the average simulated standard deviation of the multiplier distribution. Due to the small mean absolute differences in column (3), for both output and direct and indirect income multipliers, it can be concluded that the shortcut formulas approximate the true means of their respective multiplier distributions. 
Table 2

\author{
A Comparison of Shortcut Multiplier Formulas \\ for the Netherlands Interregional Model
}

\section{Output Multiplier}

(1)

\section{Column Row \\ Region to Region}

$\begin{array}{ll}1 & 1 \\ 1 & 2 \\ 1 & 3 \\ 2 & 1 \\ 2 & 2 \\ 2 & 3 \\ 3 & 1 \\ 3 & 2 \\ 3 & 3\end{array}$

(1)

\section{Column Row \\ Region to Region}

(2)

Average Actual
Multiplier

1.2381

.0315

.0306

.1768

1.4134

.2476

.0247

.0385

1.1642
(3)

Mean

$\underline{\text { Absolute Difference }}$

.0200

.0034

.0044

.0146

.0346

.0276

.0023

.0035

.0106
(4)

Mean Absolute \% Difference

$1.26 \%$

$10.67 \%$

$15.60 \%$

$7.42 \%$

$1.96 \%$

$8.89 \%$

$8.05 \%$

$7.83 \%$

$0.80 \%$

Direct and Indirect Income Multiplier

(2)

Average Actual

Multiplier
.2982
.0076
.0074
.0454
.3624
.0635
.0060
.0094
.2835

(3)

Mean

Absolute Difference

.0135

.0025

.0027

.0077

.0227

.0117

.0023

.0032

.0100
(4)

Mean Absolute $\%$ Difference

$7.34 \%$

$36.88 \%$

$46.39 \%$

$18.08 \%$

$8.12 \%$

$18.78 \%$

$71.94 \%$

$56.82 \%$

$4.36 \%$
Additionally, the small average simulation standard deviations confirm the relatively negligible dispersion in multiplier values over all random matrices with the fixed submatrix column totals. Therefore, the mean of these multiplier distributions, effectively estimated by the shortcut formulas derived in this paper, will closely estimate the actual multiplier values for the region with a very high probability.

\section{Conclusion}

Based on the empirical results, the shortcut formulas can be used to estimate interregional output, income and employment multipliers with a relatively high degree of accuracy. Although these shortcut formulas do not substitute completely for a survey 
Table 3

Netherland Interregional Model - Simulation Results

Output Multiplier

(1)

$\begin{array}{lr}\begin{array}{lr}\text { Column } \\ \text { Region to Region }\end{array} & \begin{array}{r}\text { Row } \\ 1\end{array} \\ 1 & 1 \\ 1 & 2 \\ 2 & 3 \\ 2 & 1 \\ 2 & 2 \\ 3 & 3 \\ 3 & 1 \\ 3 & 2\end{array}$

(1)

Column Row

Region to Region

$\begin{array}{ll}1 & 1 \\ 1 & 2 \\ 1 & 3 \\ 2 & 1 \\ 2 & 2 \\ 2 & 3 \\ 3 & 1 \\ 3 & 2 \\ 3 & 3\end{array}$

(2)

Average Actual

Multiplier

1.2381

.0315

.0306

.1768

1.4134

.2476

.0247

.0385

1.1642
(3)

Mean

Absolute Difference

.0004

.0002

.0001

.0003

.0006

.0005

.0000

.0001

.0002
(4)

Mean Absolute \% Difference

.0073

.0029

.0023

.0065

.0141

.0101

.0006

.0011

.0027

Direct and Indirect Income Multiplier

(2)

Average Actual

Multiplier

.2982

.0076

.0074

.0454

.3624

.0635

.0060

.0094

.2835
(3)

Mean

Absolute Difference

.0002

.0001

.0000

.0001

.0004

.0003

.0000

.0000

.0002
(4)

Mean Absolute \% Difference

.0044

.0008

.0007

.0029

.0078

.0044

.0004

.0007

.0038 based interregional model, they provide specific and accurate measures of the respective overall economic effects with only information on the direct impacts and the average effects within each regional submatrix.

Jensen and Hewings (1985), in their "requiem" to shortcut multipliers, state the following about shortcut approaches for interregional models. The BK logic would be equivalent to the claim that in input-output studies of interregional or international trade, only local propensities to consume are needed to describe satisfactorily mutual trading links.
Although we do not know their basis for this conclusion, we do not support this position or agree with the supposed equivalence. In fact, the development of shortcut formulas for interregional models in this paper draws on column total information from both the regional and interregional submatrices which reflect gross expenditure patterns both within and outside the region. We believe that this procedure represents a minimal level of detail to generate highly accurate estimates of both the regional and interregional multiplier effects. 


\section{REFERENCES}

Billings R. B. and Joseph L. Katz. "Small Area Impact Multiplier Estimates Without an Input-Output Model: A Case Study." The Southwestern Review of Business and Economics. 5 (1986). 31-41.

Burford, Roger L. and Joseph L. Katz. "Regional InputOutput Multipliers Without A Full I-O Table." Annals of Regional Science. 11 (1977). 1-26. and "A Method For

Estimation of Input-Output Type Output Multipliers When No I-O Model Exists," Journal of Regional Science, 21 (1981). 151-161. and "Shortcut InputOutput' Multipliers, Alive and Well: Response to Jensen and Hewings." Environment and Planning A. 17 (1985). 1541-1549.

DiPasquale, Denice and Karen R. Polenske. "Output, Income and Employment Input-Output Multipliers." Economic Impact Analysis - Methodology and Applications. Dordrecht Netherlands: Martinus Nijhoff Publishing, 1980.

Gamick, D. H. "Disaggregated Basic - Service Models and Regional Input-Output Models in Multiregional Projections." Journal of Regional Science. 9 (1969). 87-100.

Isard, Walter. "Interregional and Regional Input-Output Analysis, A Model of The Space Economy." Review of Economics and Statistics. 33 (1951). 318-328. "Regional Commodity Balances and Interregional Commodity Flows." American Economic Review Papers and Proceedings. 43 (1953). 167-180.

Harrigan Frank J. "The Estimation of Input-Output Type Output Multipliers When No Input-Output Model Exists: A Comment." Journal of Regional Science. 22 (1982). 375-381.

Jelavich, Mark S. "Economic Impact of Potential New Industries in a Four State Area." Annals of Regional Science. 18 (1984). 77-80.

Jensen R. C. and G. J. D. Hewings. "Shortcut 'InputOutput Multipliers: A Requiem." Environment and Planning A. 17 (1985). 747-759.

Katz, Joseph L. and Roger L. Burford. "A Comparison of Estimators of Output Multipliers from Incomplete Input-Output Data." Annals of Regional Science. 15 (1981). 39-54. and "The Random Matrix Approach to Estimation of Input-Output Multipliers." presented at the 28th North American Meeting of the Regional Science Association, Montreal. (November, 1981). a and "A Method For

Estimation of Input-Output Multipliers When No I-O Model Exists: Reply." Journal of Regional Science. 22 (1982). 382-387. and "Shortcut Multiplier Formulas for Output Income and Employment Multipliers." Annals of Regional Science. 19 (1985). 59-75.

Kuenne, R. E. "The Interregional Input-Output Model As a Derivative of a Walrasian Multiple Point System." Paper presented to the Econometric Society. (December, 1953).

Leontief, W. and A. Strout. "Multiregional Input-Output Analysis." Proceedings of an International Conference on Input-Output Techniques Geneva. (1963).

Miemyk, William. "Regional and Interregional InputOutput Models: A Reappraisal." Spatial Regional and Population Economics. New York, N.Y.: Gordon and Breach, 1963.

Miller, Ronald E. "Interregional Feedbacks Input-Output Models: Some Experimental Results." Western Economic Journal. 7 (1969). 41-50. and P. Blair. Input-Output Analysis:

Foundations and Extensions. Englewood Cliffs, N.J.: Prentice Hall, 1985.

Moses, L. N. "The Stability of Interregional Trading Patterns and Input-Output Analysis." American Economic Review. 45 (1955). 803-832.

Oosterhaven, Jan. Interregional Input-Output Analysis and Dutch Regional Policy Problems. London, Great Britain: Gower Publishers. 1981.

Phibbs, P. J. and A. J. Holsman. "An Evaluation of The Burford-Katz Short-Cut Technique for Deriving InputOutput Multipliers." Annals of Regional Science. 15 (1981). 11-19.

Polenske, Karen. "An Emperical Test of Interregional Input-Output Models: Estimation of 1963 Japansese Production." American Economic Review. 60 (1970). 26-82. Multiregional Input-Output Analysis. Lexington, MA.: Lexington Books D. C. Heath, Inc., 1972.

- "The Implementation of a Multi-

Regional Input-Output Model for the United States." Input-Output Techniques. London, England: North Holland, 1972. a . State Estimates of Technology. Lexington, MA.: Lexington Books, D. C. Heath, Inc., 1974.

"Multiregional Interactions Between Energy and Transportation." Advances In InputOutput Analysis. Cambridge, MA: Ballinger Publishing Co., 1976. 
"Energy Analysis and the

Determination of Multi-Regional Prices." Paper presented at the 25th North American Meetings of the Regional Science Association, Chicago.

(November, 1978). . "Construction of the U.S. Multigeneral Input-Output Accounts." Department of Urban Studies and Planning Massachusetts Institute of Technology working paper. (November, 1979).

Richardson, Harry W. "Input-Output and Economic Base Multipliers: Looking Backward and Forward." Journal of Regional Science. 25 (1985). 607-661.

Round, Jeffrey I. "On Estimating Trade Flows In Interregional Input-Output Models." Regional Science and Urban Economics. 8 (1978). 289-302.
"Compensating Feedbacks In Interregional Input-Output Models." Journal of Regional Science. 19 (1979). 145-155. . "Nonsurvey Techniques: A Critical Review of the Theory and the Evidence." International Regional Science Review. 8 (1983). 189-212.

Stein, J. L. "Interregional Comparisons of the Marginal Product of Capital." Southern Economic Journal. 25 (1958). 24-32.

Tiebout, C. M. "Regional and Interregional Input-Output Models: An Appraisal." Southern Economic Journal. 24 (1957).

West, G. R., J. B. Morrison, and R. C. Jensen. An Interregional Input-Output Table for Queensland. Department of Economics, University of Queensland, October 1982. 
